\title{
Diagnostic dyspraxia by disrupted fiber connections of the posterior corpus callosum after distal anterior cerebral artery aneurysm rupture
}

\author{
Giulia Cossu • Mahmoud Messerer • \\ Roy Thomas Daniel • Karin Diserens
}

Received: 24 March 2014 / Accepted: 16 April 2014 / Published online: 6 May 2014

(C) Springer-Verlag Wien 2014

\section{Dear Editor,}

We would like to report a well-documented case of diagnostic dyspraxia as a consequence of a ruptured distal anterior cerebral artery (DACA) aneurysm. Radiological findings on tractography permit a better understanding of the physiopathology and anatomo-clinical correlations between diagnostic dyspraxia and intracranial hemorrhage secondary to a DACA aneurysm rupture.

A right-handed patient presented with brutal onset of left temporal headache. Clinical examination revealed a Glasgow Coma Scale of 14 with a right crural hemiparesis. The cerebral CT scan showed a subarachnoid hemorrhage Fisher grade IV (Fig. 1a), with the majority of the blood in the pericallosal cistern. The angio-CT demonstrated a right A2 aneurysm at the pericallosal - callosomarginal junction; this was treated immediately by urgent endovascular coiling with no periprocedural complications.

On day 1, the patient complained about the feeling of oppositional behavior from his non-dominant hand against his dominant hand. The left hand-dissociative movements were triggered by voluntary activities of his right hand: when we asked the patient to take an object with his right hand, his left hand seized the object before the right hand had time to

G. Cossu $(\bowtie) \cdot$ M. Messerer $\cdot$ R. T. Daniel

Département des Neurosciences Cliniques, Service de

Neurochirurgie, Centre Hospitalier Universitaire Vaudois, Université

de Médecine et Biologie de Lausanne, Rue du Bugnon 44,

1011 Lausanne, Switzerland

e-mail: giulia.cossu@chuv.ch

\section{K. Diserens}

Département des Neurosciences Cliniques, Unité de

Neurorééducation Aiguë et Neurorééducation des Maladies

Neurodéveloppementales Adultes et Jeunes Adultes, Service de

Neurologie, Centre Hospitalier Universitaire Vaudois, Université de Médecine et Biologie de Lausanne, Rue du Bugnon 44,

Lausanne 1011, Switzerland reach it. The patient never doubted that the left hand was part of his own body, and he had no left/right hand confusion, but he felt that his left hand had its own will. No frontal release signs such as grasping or perseverance were observed. Neuropsychological testing showed language difficulties and clinical signs of callosal disconnection (bilateral taping disturbance, interdigital transfer difficulties, left tactile extinction) associated with executive dysfunction.

In the subacute phase, T2- and DWI-weighted MRI images showed involvement of the body and splenium of the corpus callosum with residual blood in the pericallosal cistern (Fig. 1b). The tractographic analysis confirmed the interhemispheric disconnection at the posterior third of the corpus callosum with a complete interruption of the commissural fibers (Fig. 1c, d).

The patient underwent a specialized rehabilitation and had a good recovery with a complete return to autonomous daily life activities.

DACA aneurysms account for about 3 to $7 \%$ of intracranial aneurysms, most of which commonly arise at the bifurcation of the pericallosal and calloso-marginal arteries [3]. Ruptured DACA aneurysms are smaller than in other sites and are associated with more frequent intracerebral hematomas [4].

The Alien Hand Syndrome (AHS) was first described by Goldstein as a variety of clinical conditions whose common characteristic is the uncontrolled behavior or the feeling of strangeness of one extremity, most frequently involving the left hand $[1,2]$.

The common classification distinguishes between the posterior, or sensory form, of AHS and the anterior, or motor form, of this condition. To explain inconsistencies such as the phenomenon of diagonistic dyspraxia, Aboitiz (2003) proposed the distinction of five classes of AHS with peculiar clinical manifestations and specific anatomical substrates [1]. 

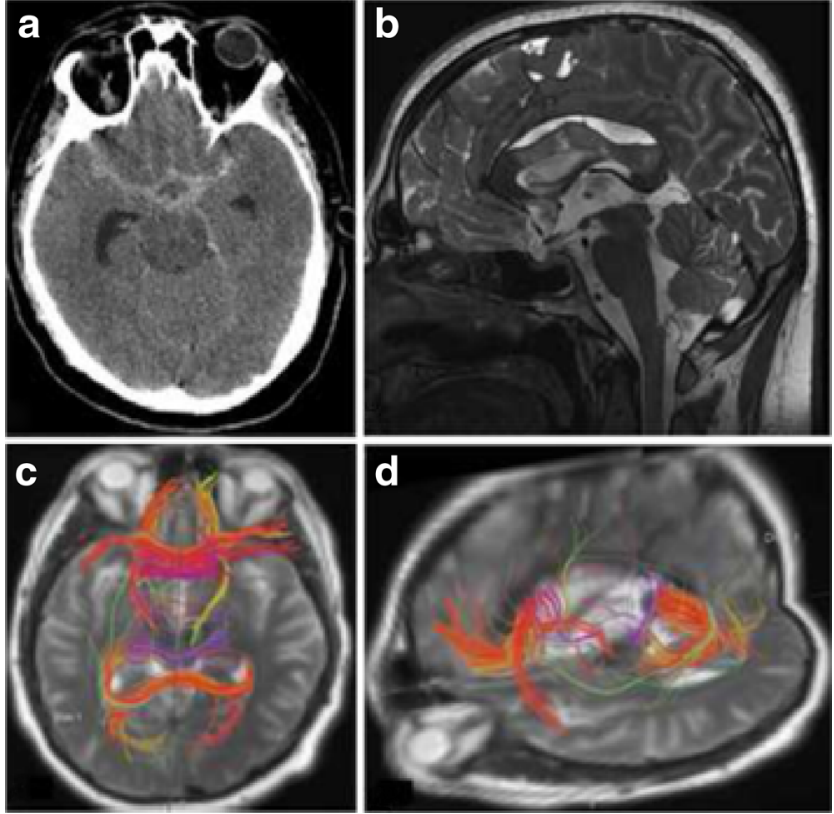

Fig. 1 Axial view of cerebral CT scan showing a subarachnoid hemorrhage Fisher IV from a ruptured DACA aneurysm (a). Sagittal T2-weighted MRI images in the subacute phase showing involvement of the posterior body and splenium of the corpus callosum with residual blood in the pericallosal cistern (b). Tractographic assessment by diffusion tensor imaging (DTI) has been used to visualize commissural fibers. Axial T2-weighted MRI image with a tractographic reconstruction of interhemispheric connections (c). Sagittal T2-weighted MRI image showing the inter-hemispheric disconnection at the posterior third of the corpus callosum with an interruption of commissural fibers (d)

Diagonistic dyspraxia was first described in callosotomized patients $[1,2]$ as a clinical manifestation in which the left hand (in right-handed subjects) performs actions contrary or opposite to, or interferes with, the actions executed by the right hand. Lesions in the posterior end of the body of the corpus callosum, especially in its ventral part, seem to be responsible for this peculiar manifestation $[5,8,9]$.

Tanaka et al. [9] defined this syndrome as a "peculiar dissociative behavior of the left hand in the absence of pathological grasping phenomena: the left hand often acted at cross-purposes to the right". Parkin proposed that normally the hemisphere ipsilateral to the intended hand is inhibited by the contralateral hemisphere via the corpus callosum [7]; in patients with callosal damage, this contralateral control is absent, and the ipsilateral hemisphere becomes engaged in the task, thus generating an intermanual conflict.

Our patient presented with a typical clinical manifestation of diagnostic dyspraxia, and the lesions were predominantly localized in the posterior half of the callosal body and had no significant cortical involvement, as in the three cases reported from Nishikawa [6].

We report here the first case of diagnostic dyspraxia with clear tractographic images of fiber disruption in the posterior mid-body and splenium of the corpus callosum due to a ruptured aneurysm of the distal anterior cerebral artery.

Different pathophysiological mechanisms underlie the diverse behaviors in AHS. Our results confirm the hypothesis that the motor disturbance in diagnostic dyspraxia is attributable to the lack of contralateral inhibition of the intended hand, a condition present with normal motor behavior during bimanual tasks.

However, further studies are needed to elucidate the brain regions and the cerebral mechanisms involved in this process.

Acknowledgments We would like to acknowledge Prof. Philippe Maeder, Mr Timothy Montoute and Dr Benjamin Richoz for their help and advices.

Conflicts of interest None.

\section{References}

1. Aboitiz F, Carrasco X, Schroter C, Zaidel D, Zaidel E, Lavados M (2003) The alien hand syndrome: classification of forms reported and discussion of a new condition. Neurol Sci 24(4):252-257

2. Akelaitis AJ, Risteen W, Herren R, Van Wagenen W (1942) Studies of the corpus callosum. III. A contribution to the study of dyspraxia and apraxia following partial and complete section of the corpus callosum. Arch Neurol Psychiatr 47:971-1008

3. De Sousa AA, Dantas FL, de Cardoso GT, Costa BS (1999) Distal anterior cerebral artery aneurysms. Surg Neurol 52(2):128-135, discussion 135-6

4. Fein JM, Rovit RL (1970) Interhemispheric subdural hematoma secondary to hemorrhage from a calloso-marginal artery aneurysm. Neuroradiology 1(3):183-186

5. Feinberg TE, Schindler RJ, Flanagan NG, Haber LD (1992) Two alien hand syndromes. Neurology 42(1):19-24

6. Nishikawa T, Okuda J, Mizuta I, Ohno K, Jamshidi J, Tokunaga H, Ikejiri Y, Nakagawa Y, Yoshimine T, Tanabe H, Takeda M (2001) Conflict of intentions due to callosal disconnection. J Neurol Neurosurg Psychiatry 71(4):462-471

7. Parkin A, Barry C (1991) Alien hand sign and other cognitive deficits following ruptured aneurysm of the anterior communicating artery. Behav Neurol 4:167-179

8. Suwanwela NC, Leelacheavasit N (2002) Isolated corpus callosal infarction secondary to pericallosal artery disease presenting as alien hand syndrome. J Neurol Neurosurg Psychiatry 72(4):533-536

9. Tanaka Y, Yoshida A, Kawahata N, Hashimoto R, Obayashi T (1996) Diagonistic dyspraxia. Clinical characteristics, responsible lesion and possible underlying mechanism. Brain 119(Pt 3):859-873 